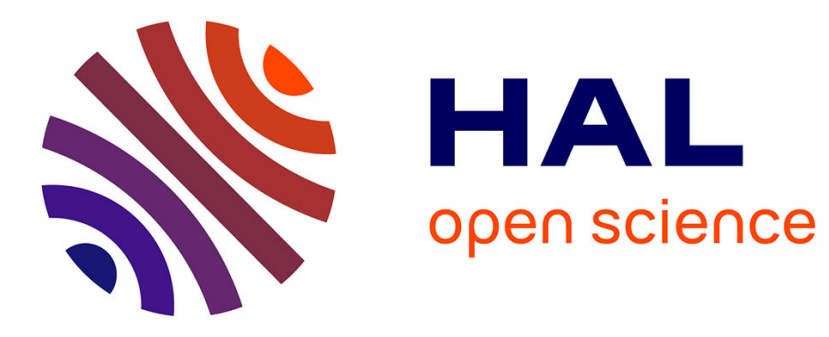

\title{
Figuration, fable et théologie dans les traités de mythographie
}

\author{
Rachel Darmon
}

\section{To cite this version:}

Rachel Darmon. Figuration, fable et théologie dans les traités de mythographie. Réforme, Humanisme, Renaissance, 2013, 77, pp.31-50. 10.3406/rhren.2013.3327 . halshs-01462976

\section{HAL Id: halshs-01462976 \\ https://shs.hal.science/halshs-01462976}

Submitted on 10 Feb 2017

HAL is a multi-disciplinary open access archive for the deposit and dissemination of scientific research documents, whether they are published or not. The documents may come from teaching and research institutions in France or abroad, or from public or private research centers.
L'archive ouverte pluridisciplinaire HAL, est destinée au dépôt et à la diffusion de documents scientifiques de niveau recherche, publiés ou non, émanant des établissements d'enseignement et de recherche français ou étrangers, des laboratoires publics ou privés. 
Figuration, fable et théologie dans les traités de mythographie Rachel Darmon

\section{Citer ce document / Cite this document :}

Darmon Rachel. Figuration, fable et théologie dans les traités de mythographie. In: Réforme, Humanisme, Renaissance, n77, 2013. pp. 31-50;

doi : 10.3406/rhren.2013.3327

http://www.persee.fr/doc/rhren_1771-1347_2013_num_77_1_3327

Document généré le 24/01/2017 


\title{
Figuration, fable et théologie dans les traités de mythographie
}

\author{
Rachel DARMON \\ Université Paris VIII
}

Figura est le titre qu'Auerbach a donné à son essai sur la Divine Comédie de Dante à partir de laquelle il tente de définir les fondements de l'herméneutique médiévale ${ }^{1}$. Il assume un anachronisme critique en proposant de distinguer, à l'encontre des usages de lépoque étudiée, figure et allégorie. La notion de figure lui semble en effet seule capable d'expliquer ce qui fait la spécificité de la littérature chrétienne antique et médiévale. Lallégorie serait un concept opératoire pour le registre profane, tandis que la notion de figure permettrait de rendre compte de ce qui, dans la littérature, est propre à l'univers de pensée chrétien. Ce système de représentation inscrirait les êtres dans la superposition d'une temporalité double: celle d’avant et celle d’après le Jugement Dernier. En conséquence, la notion de figure rendrait compte du fait que les êtres et les choses, dans la Divine Comédie et plus généralement dans la littérature médiévale, renvoient à la fois et dans le même temps à quelque chose de concret et d'abstrait, au passé et au futur, à l'ici-bas et à l'au-delà.

Cest donc une théologie proprement chrétienne qui est, selon Auerbach, à lorigine du régime de figuration médiéval. Qu’en est-il lorsque ce régime se trouve confronté à une théologie païenne, lorsqu'il cherche dans l'antique mythologie la source d'une poésie le rapprochant, paradoxalement, du Dieu chrétien?

1. Erich Auerbach, Figura, traduit de l'allemand par Marc Henri Bernier, Paris, Belin, 1993. Première publication dans Neue Dantestudien, Istambul, Basimevi, 1944. 
Boccace inscrit avec insistance ses pas dans ceux de Dante. À la charnière du Moyen Âge et de la Renaissance, il fonde sa défense de la poésie précisément sur un réexamen des notions de fable, de figure et d'allégorie. Ces considérations théoriques constituent l'introduction (préface ou prohemium) et la conclusion (deux derniers des quinze livres) d'une immense somme mythographique, la Genealogia deorum gentilium. Cet ouvrage sert de source d'information et d'inspiration aux peintres et aux lettrés de toute la première Renaissance, jusquà ce qu'une série d'autres mythographies, publiées dans un intervalle de temps très resserré (entre 1532 et 1567), ne vienne la renouveler. Dans la Genealogia deorum de Boccace, il y a donc un lien intrinsèque entre le statut de la fiction, désigné par le terme de fable, et ce qu’on appelle souvent « la fable ", désignant, lorsqu’elle est précédée en français de l’article défini, la mythologie des anciens. Le terme de fable, en français comme en latin, comporte effectivement une ambiguïté, que Conti ${ }^{2}$, entre autres, se plaît à rappeler: fable peut désigner aussi bien les mythes des Anciens que les fables d'Esope ou les contes de vieille femme. Faut-il alors distinguer des autres fables celles qui retracent l'histoire des dieux antiques? La fable mythologique est-elle une fiction comme les autres, ou bien son statut d'ancienne théologie lui confère-t-elle un statut spécifique? Je propose d'examiner dans cet article en quoi les mythographies, ou études spécialement consacrées par des humanistes à la mythologie antique, sont loccasion d'un réexamen des notions de fable et de figuration.

Afin de clarifier les termes de l'analyse, je commencerai par rappeler la manière dont Boccace s'approprie les termes de fable et de figure dans sa Genealogia deorum: il s’appuie sur l'héritage du système herméneutique médiéval pour mieux le modifier, au profit d'une valorisation de la poésie profane. Le pivot de ce changement consiste en une réévaluation des discours sur le divin: la notion de théologie est au cœur de cette transformation. J'analyserai enfin la manière dont les mythographes du

2. Natale Conti, Mythologiae, siue Explicationum fabularum libri decem, Venise, Comin da Trino, 1567, ch. 3, "De fabularum uarietate » et ch. 4, " De apologorum, fabularum, aenorumque differentia ", p. 5-8 dans l'édition d'André Wechel, Francfort, 1584. 
$\mathrm{XVI}^{\mathrm{e}}$ siècle, de Pictorius à Conti, se réapproprient cette notion pour déplacer le questionnement de la figure aux enjeux de la figuration.

\section{Fable et figure, deux aspects d'un même langage}

Quel rapport Boccace établit-il entre fable et figure? Le terme de fable renvoie à l'art du discours, tandis que celui de figure est associé, de nos jours, à une représentation concrète, picturale. Cette opposition entre le verbal et le pictural n'a pas cours chez Boccace. Fable et figure renvoient toutes deux à un matériau textuel. Toutes deux sont le résultat d'un travail de façonnement, délaboration, qui vise à donner aux vérités qu'elles contiennent une apparence plaisante. Il s'agit alors pour l'interprète de dépasser cette apparence des fables et des figures pour accéder au véritable sens. Boccace reprend sur ce point la tradition antique et médiévale de l'allégorie, qui invite à enlever l'écorce que constitue le sens premier pour parvenir au sens profond que l'auteur a mis dans son œuvre. Le livre XIV de sa Genealogia deorum gentilium donne la définition suivante:

Fabula est exemplaris seu demonstratiua sub figmento locutio, cuius amoto cortice, patet intentio fabulantis ${ }^{3}$.

La fable est une manière de parler par fiction, exemplaire ou démonstrative. Une fois qu'on en enlève lécorce, on découvre l'intention de celui qui parle 4 .

Ce qui caractérise la fable ici est le mot « figmentum », qui dérive, tout comme figura, du verbe fingere: fable et figure sont des modes d'expression non spontanés, qui nécessitent un travail de modelage, de façonnage. Boccace utilise, comme en français moderne, l’expression de « figures du

3. Boccace, Genealogia deorum gentilium, XIV, 9. J'ai consulté, pour l'établissement du texte latin des livres I à V, l'édition de Jon Solomon, The I Tatti Renaissance Library, Harvard University Press, Cambridge et Londres, 2011, et, pour les autres livres, lédition de Vittore Branca, Tutte le opere di Giovanni Boccaccio, vol. 7 et 8, Milan, A. Mondadori, 1998.

4. Sauf mention contraire, toutes les traductions sont miennes. 
discours ${ }^{5} »$. Il s'agit d'un travail d'élaboration sur la matière verbale ${ }^{6}$, qui s'oppose à l'expression directe de concepts abstraits comme le fait par exemple, le discours des philosophes scolastiques. Fable et figure correspondent à un mode de signification indirect qui appelle déchiffrement. Tous deux sont des signifiants, qui renvoient vers un ou plusieurs signifiés.

Toutefois, la figure peut consister en une belle apparence aussi bien qu'en un signe abstrait. Dans la notice qu'il consacre à Minerve, Boccace emploie le terme de figura pour désigner les chiffres mathématiques:

Illi insuper Titus Livius attribuit numerorum inuentionem et eorumdem figuras, cum ante loco numeri signis uterentur antiqui?

Tite-Live de plus lui attribue l'invention des nombres et la manière de les figurer, puisquauparavant les anciens usaient de signes à la place du nombre.

Le terme figura désigne donc ici un signifiant très sobre, qui renvoie à une abstraction mathématique. Dans la notice sur Pan, le même terme figura renvoie au contraire à l'apparence complexe et chatoyante d'une divinité mythologique.

Huius preterea poete et alii insignes viri mirabilem descripsere figuram. (...) Is ante alia fronti habet infixa cornua in celum tendentia,

Barbam prolixam (...)

Preterea inferioribus membris hirsutum atque hispidum dicit, et pedes habere capreos (....). His igitur premissis, ad intrinseca veniendum est ${ }^{8}$. De plus, des poètes et autres hommes illustres ont décrit son étonnante figure $(\ldots)$

«Avant toute chose il avait des cornes fixées sur le front, tendues vers le ciel, Une barbe abondante (...)

5. Boccace, Genealogia deorum gentilium, XIV, 12: "Si forsan uelint duriciem textus, figuras dictionum, aut orationum colores, et peregrinorum uocabulorum incognitam a se pulchritudinem damnasse. "

6. Cf. Quintilien, Institution oratoire, IX, I, 10-13: «Quare primum intuendum est quid accipere debeamus figuram. Nam duobus modis dicitur: uno qualiscumque forma sententiae, sicut in corporibus, quibus, quoquo modo sunt composita, utique habitus est aliquis: altero, quo proprie schema dicitur, in sensu vel sermone aliqua a vulgari et simplici specie cum ratione mutatio. (...) Sed si habitus quidam et quasi gestus sic appellandi sunt, id demum hoc loco accipi schema oportebit quod sit a simplici atque in promptu posito dicendi modo poetice vel oratorie mutatum."

7. Boccace, Genealogia deorum gentilium, II, 3, 2-3.

8. Boccace, Genealogia deorum gentilium, I, 4, 2 sq. 
Il [le poète] dit de plus qu'il avait les membres inférieurs hirsutes et velus, ainsi que des pieds de chèvre. (...) Après ces préliminaires, il faut en venir à ce qui est au-dedans.

Figura désigne ici l'apparence de Pan. Elle renvoie à un corps composite, mêlant cornes, barbe, poils et pieds de chèvre. Le truculent dieu mythologique comme le chiffre mathématique sont tous deux désignés par le même terme de figure : tous deux appellent un signifié qui n'est pas directement donné. Ils ne sont qu'une apparence qu’il s'agit de dépasser (« ad intrinseca ueniendum est»). Un peu plus loin, au livre X, Boccace explique que Pégase est la «figure » de la renommée? . Le mot «figure » désigne donc tout élément appelant une interprétation, associant à une apparence extérieure une autre signification. La notion de fable est intimement liée à celle de figure: toutes deux renvoient au processus de figuration.

La fable ne correspond pas nécessairement à une narration. Pour Boccace, comme pour les mythographes du $\mathrm{XVI}^{\mathrm{e}}$ siècle, étudier les fables des Anciens ne signifie pas raconter un récit mythologique, mais interpréter chaque figure, sans qu'un fil narratif ne les relie nécessairement entre elles. L'intérêt de la fable mythologique, pour ces auteurs, ne repose pas dans la narration, ni dans l'agencement d'un récit, mais dans la succession de figures juxtaposées. On peut dire que la fable consiste, dans cette perspective, en un assemblage de figures. La notion de figure désigne une forme condensée, ramassée, dont l'efficacité repose sur la densité. Cette condensation sémantique peut ensuite être explicitée, développée, déployée dans un discours explicatif. En 1532, l'humaniste germanique Georgius Pictorius consacre à son tour un traité spécifique à la mythologie ${ }^{10}$. Louvrage ne comporte aucune narration, mais est composé d'une série de chapitres, chacun consacré à un dieu. Chaque chapitre est toujours divisé en trois parties : la première énumère et explicite les différents noms du dieu; la deuxième décrit son apparence, en employant indifféremment les termes de " simulacrum », « imago ", " effigies », " pictura »;

9. Boccace, Genealogia deorum gentilium, X, 27: «Dicit ergo Pegasum ex Meduse sanguine nasci, eo quod in figura fame constitutus sit. "

10. Pictorius, Theologia mythologica, Fribourg-en-Brisgau, Johannes Faber (Emmeus), 1532. 
la troisième développe les interprétations allégoriques liées à cette description. Chaque dieu constitue ainsi une figure à déchiffrer. Dans sa préface, Pictorius désigne bien l'objet du traité par le terme de «fabula», dont il cherche à montrer l'utilité. La mythologie est une fable, non au sens de narration ou de "récit des origines ", comme on la définit souvent actuellement, mais au sens de fiction, de langage figuré. Chaque nom, chaque détail ekphrastique donnent matière à interprétation. L'ensemble de ces détails forme la figure globale de chaque dieu étudié. La mythographie ainsi comprise ne raconte pas des mythes, mais présente des figures composites, formées à partir de fragments signifiants épars. Les traités qui se développent très rapidement dans les années suivant la publication de celui de Pictorius appréhendent la mythologie de la même façon. Le De deis gentium uaria et multiplex historia de Giraldi (Bâle, 1548), les Imagini de i Dei de Cartari (Venise, 1556), les Mythologiae, siue fabularum explicationum libri de Conti (Venise, 1567) présentent une succession d’anciennes divinités: le mythographe expose différentes informations concernant le dieu, entrelardées de citations poétiques ou de propos en prose empruntés à des auteurs antiques, médiévaux ou modernes. Ces citations au sujet des figures divines appellent, à leur tour, d'autres interprétations, dans un système herméneutique où on explique la figure par une autre figure, qui en appelle une autre encore. Pour expliquer tel aspect de la description d'un dieu, on cite un poème antique; cette citation devient elle-même une figure qui demande à son tour à être interprétée, par comparaison avec une autre citation ou information, et ce à l'infini. Chaque figure renvoie à une multitude d'autres, puisque le principe de la figuration est l'association déléments d’ordres différents.

On peut ainsi expliquer un texte par une image, ou une image par un texte. Les figures peuvent être picturales ou verbales, apparaître concrètement ou non sur la page du livre. Chez Boccace, comme chez la plupart des mythographes du XVI ${ }^{\mathrm{e}}$ siècle, les figures des dieux païens sont faites de mots. Il s'agit de descriptions, dont le détail est souvent emprunté à plusieurs auteurs différents. Dans l'extrait de la Genealogia deorum gentilium de Boccace cité ci-dessus, la « figure » de Pan est décrite au moyen de 
citations empruntées à Raban Maur et à Virgile. Dans la seconde mythographie de Pictorius, intitulée Apotheoseos deorum libri ${ }^{11}$, le terme figura désigne en revanche le plus souvent une image imprimée. La description de la déesse Junon, par exemple, s'achève par la formule « exemplo haec sequens figura esse poterit ${ }^{12}$ » («la figure ci-dessous peut en être un exemple »), suivie immédiatement de l'impression d'un bois gravé représentant Junon. Le terme figura désigne donc ici une illustration, une figure picturale qui s'inscrit dans la matérialité de la page. Cette figura est elle aussi mystérieuse et suscite l'interprétation. Texte et image constituent l'un comme l'autre des figures: ce qui compte dans cette conception de la figure nest pas la nature (verbale ou picturale) du signifiant, mais le processus de signification dont il est loccasion.

On comprend mieux, dès lors, le régime de l'image dans les mythographies du Xvi ${ }^{\mathrm{e}}$ siècle. La plupart d'entre elles annoncent par leur soustitre la présence d'images, alors que l'ouvrage n'est pas illustré. La Theologia mythologica de Pictorius propose, dans chaque chapitre, une rubrique intitulée " image » (« imago » ou " effigies»), qui consiste en une description. Le De Deis gentium historia, in qua simul de eorum imaginibus et cognominibus agitur (Histoire des dieux, où l'on traite à la fois de leurs images et de leurs noms) de Giraldi emprunte à Pausanias de nombreuses descriptions de statues, mais ne comporte pas non plus d'illustration. Enfin, les fameuses Imagini de i Dei (Images des dieux) de Cartari ne sont pas illustrées dans leurs premières éditions: l'ajout d'images imprimées sur la page est postérieur de quinze ans à l'editio princeps. Dans chacun de ces ouvrages, les images annoncées consistent en des descriptions qui appellent l'interprétation. L'image ou la figure désignent moins une réalité picturale qu'un mode d'exploration. Elles sont une des nombreuses formes que peut emprunter la fiction.

11. Pictorius, Apotheoseos tam exterarum gentium quam romanorum deorum libri tres, Bâle, Nikolaus Brylinger, 1558. Il existe deux émissions de ce texte avec modification du titre, que j’ai commentée dans ma thèse: Rachel Darmon, Dieux futiles, dieux utiles. Lécriture mythographique et ses enjeux dans l'Europe de la Renaissance. Autour des traités de Georgius Pictorius (1500-1569), thèse de doctorat soutenue le 13 décembre 2012 à l'Université Paris VIII, sous la direction de F. Graziani.

12. Pictorius, Apotheoseos deorum libri, p. 17. 


\section{Figuration et discours théologique}

C'est précisément l'apport de Boccace que d'avoir élaboré une argumentation très serrée démontrant l'importance du langage figuré1 ${ }^{13}$. Dans les deux derniers livres de sa Genealogia, il montre que ce mode d'expression détournée a son intérêt en tant que tel. Il n'est certes pas le premier à défendre l'intérêt des fables au moyen de l'allégorie ${ }^{14}$, mais son argumentation est particulièrement polémique. Elle prend le contre-pied de la tradition dominante. Yves Delègue ${ }^{15}$ rappelle que dans la tradition médiévale, l'image est suspectée dêtre mensonge et séduction. La fable, qui use de figures et images verbales, est ainsi condamnée, à moins qu'elle ne soit interprétée en un sens moralisé (comme c'est le cas avec les Métamorphoses d'Ovide) ou selon la quadruple exégèse appliquée d’ordinaire aux Saintes Écritures (ce qui ne va pas sans susciter certaines contestations). Ce type d'interprétation confère alors à la fable païenne un sens proprement chrétien. Dans cette perspective, c'est moins la fable ou la figure que le résultat de l'interprétation qui est important. Lécorce n’est qu'une enveloppe amusante et futile, dont le seul intérêt est de mener plaisamment à la vérité. Yves Delègue rappelle que la poésie n’a pas le statut d’art libéral ${ }^{16}$, à la différence de la philosophie et de la théologie qui offrent un accès direct et sans détour à la vérité. Pour Boccace au contraire, parler par figures est une nécessité. Il rappelle les propos de Denys l'Aréopagite selon lesquels "la divine théologie use de fictions poétiques ${ }^{17}$ »; Jésus lui-même use de

13. Françoise Graziani a consacré de nombreux travaux à ce sujet, notamment La Pensée fossile: mythe et poésie d'Aristote à Vico, thèse pour obtenir le grade de docteur d'État, sous la direction de Marc Fumaroli, soutenue le 20 novembre 2010 à l'Université Paris IV et « La confabulation poétique selon Boccace ", in Formes, usages et visées des pratiques mythographiques de l'Antiquité à la Renaissance, Actes du colloque Polymnia tenu à Lille, Lyon et Genève de mai à décembre 2011, Lille, Presses universitaires du Septentrion, à paraître en 2014.

14. Cf. Jean Pépin, La Tradition de l'allégorie de Philon d'Alexandrie à Dante, Paris, Études Augustiniennes, 1987.

15. Yves Delègue, Traduction, présentation et annotation à Giovanni Boccaccio, La Généalogie des dieux païens, Livres XIV et XV, Strasbourg, Presses universitaires de Strasbourg, 2001.

16. Yves Delègue, présentation à Giovanni Boccaccio, La Généalogie des dieux païens, Strasbourg, Presses universitaires de Strasbourg, 2001, p. 9-10.

17. Boccace, Genealogia deorum gentilium, XIV, 18: "diuinam theologiam poeticis fictionibus uti ». 
paraboles, c'est-à-dire d'un langage figuré, semblable à celui des poètes ${ }^{18}$. Boccace ne se contente pas de comparer le langage figuré du poète à celui de la théologie chrétienne. Il identifie l'un à l’autre, faisant fi de toute distinction entre profane et sacré ou, plus exactement, en assimilant la poésie à un langage sacré, inspiré, dans ses fictions mêmes, par le Dieu chrétien. Il commence par expliquer que « ce que le poète appelle fable ou fiction, nos théologiens l'ont appelé figure ${ }^{19} »$. Fable, fiction, figure sont équivalents. Il ne s'agit, suggère Boccace, que d'une différence d'appellation. Il revient sur ce point quelques paragraphes plus loin:

Preterea superaddent ab eis scripta fictiones non esse, sed potius esse figuras, et sic nuncupari debere, et per consequens figuratores eorundem scriptores. O ridendum diffugium, quasi credituri simus, quod simillimum est in cortice, mutatione seu diuersitate nominis effectus habeat diuersos efficer ${ }^{20}$ ! Ils [les détracteurs de la poésie] ajouteront que leurs écrits [ceux de Jean l'évangéliste et des prophètes chrétiens] ne sont pas des "fictions", mais plutôt des "figures ", que c'est ce terme qui convient, et que par conséquent ceux qui écrivent ainsi sont des « figurateurs». La ridicule échappatoire! Comme si nous allions croire que ce qui est absolument semblable par lécorce pouvait avoir des effets différents parce quoon a modifié ou changé son nom ${ }^{21}$ !

La différence, suggérée par le lexique, entre figure du langage sacré chrétien et fiction des poètes est illusoire, d’après Boccace. Il s’agit du même type d' "écorce ", du même langage figuré, qui produit les mêmes effets: tous deux révèlent des vérités du même ordre. La fiction, comme la théologie, permet de se rapprocher de la vérité de Dieu. Dans la préface qui ouvre la Genealogia, Boccace se représente en explorateur de la mythologie païenne guidé par le Dieu chrétien : c'est Dieu qui lui inspire

18. Boccace, Genealogia deorum gentilium, XIV, 18: «nonne ipse etiam Dominus et Saluator noster multa in parabolis locutus est, comico conuenientibus stilo? Nonne et ipse adversus Paulum prostratum Terrentii uerbo usus est, scilicet durum est tibi contra stimulum calcitrare?".

19. Boccace, Genealogia deorum gentilium, XIV, 9: « et quod poeta fabulam aut fictionem nuncupat, figuram nostri theologi uocauere" .

20. Boccace, Genealogia deorum gentilium, XIV, 13.

21. Je m’inspire en la modifiant de la traduction d'Yves Delègue, La Généalogie des dieux païens, Strasbourg, Presses universitaires de Strasbourg, 2001, p. 58. 
sa quête au cœur des fictions des Anciens ${ }^{22}$. Dans cette même préface, il reprend à son compte les propos d'Aristote, transmis par Saint Augustin: "les poètes furent les premiers théologiens ${ }^{23}$ ".

La figure n'est donc plus l'apanage des théologiens, ou, plus exactement, elle n'est plus l'apanage des théologiens chrétiens. Les poètes anciens sont les messagers de l'antique théologie, eux aussi parlent par figures, lesquelles ne doivent pas systématiquement être ramenées à un sens chrétien. Boccace propose d’assouplir les frontières un peu rigides de l'interprétation quadripartite (sens littéral, allégorique, anagogique, tropologique) pour conférer aux anciennes fables un sens plus ouvert, moins strictement encadré.

Veteres quippe, relictis licteris suis nominibus insignitis, in uiam uniuerse carnis abiere, sensusque ex eis iuxta iudicium post se liquere nascentium, quorum quot sunt capita, fere tot inueniuntur iudicia. Nec mirabile; uidemus enim diuini uoluminis uerba ab ipsa lucida, certa, ac immobili veritate prolata, etiam si aliquando tecta sint tenui figurationis uelo, in tot interpetrationes distrahi, quot ad illa deuenere lectores. Et ob id in hoc minus pauescens accedam, nam, et si minus bene dixero, saltem ad melius dicendum prudentiorem alterum excitabo. Et hoc faciens, primo, que ab antiquis hausisse potero, scribam, inde, ubi defecerint seu minus iudicio meo plane dixerint, meam apponam sententiam ${ }^{24}$.

De fait les Anciens ont laissé derrière eux leur nom rendu célèbre par ce qu'ils ont écrit et sen sont allés sur la route qui attend toute chair. Ils ont laissé l'interprétation de leurs œuvres au jugement de ceux qui naîtraient après eux, qui ont presque chacun une opinion différente. Cela n'est pas étonnant. Nous voyons en effet que les mots du livre divin, d'eux-mêmes clairs, sûrs, portés par une inébranlable vérité, même s'ils sont parfois couverts du fin voile de la figuration, sont écartelés en autant d'interprétations qu'il y a eu de lecteurs pour y recourir. Pour cette raison jaborderai ce travail avec moins d'appréhension. Car si je dis moins bien les

22. Boccace, Genealogia deorum gentilium, Préface, I, 51. J’ai développé dans ma thèse de doctorat, p. 306-309, une analyse comparative de ce passage avec certains extraits de la Divine Comédie.

23. Boccace, Genealogia, Préface, II, 4 : «poetae, quos primos theologizantes Aristotiles dicit». Voir aussi Augustin, Cité de Dieu, XVIII, 14 et Aristote, Métaphysique, 983 b.

24. Boccace, Genealogia, Préface, I, 43. 
choses, du moins aurai-je incité quelqu'un d'autre, plus sage que moi, à mieux les dire. Pour ce faire, j'écrirai d'abord ce que j’aurai pu puiser chez les Anciens; à partir de là, quand leurs écrits feront défaut ou diront moins pleinement que ce qu'il me semble bon, j’ajouterai ma propre idée.

Se trouve ici souligné l'écart qui sépare le texte de l'interprétation qu'en donnent les lecteurs. Les auteurs antiques sont morts, Dieu ne se montre pas directement: nul interprète ne peut prétendre donner une interprétation unique et définitive. Boccace prend acte de cette relativité des lectures et propose en conséquence d'en accepter la diversité. L'incapacité à retrouver un sens originel avec certitude sera donc compensée par une dynamique créatrice, proposant une multitude d'interprétations, anciennes et nouvelles. Boccace applique immédiatement cette herméneutique à son enquête mythographique. Dans un des premiers chapitres de la Genealogia, il affirme que « dans ces fictions, il n'y a pas une unique interprétation, bien au contraire, on peut dire que le sens est polysémique, c'est-à-dire multiple ». Il donne alors successivement le sens littéral ou historique, puis moral, allégorique et enfin anagogique de la victoire de Persée sur la Gorgone. Il en conclut que ces différents sens peuvent tous êtres désignés par le terme globalisant de "allégoriques », puisque le terme allégorie vient du grec allon, qui signifie simplement " autre, différent ${ }^{25}$ ». Boccace repense l'articulation entre figuration et théologie en accordant aux fables païennes le statut de figure et en les pensant partiellement sur le modèle de l'exégèse biblique. Ce modèle exégétique, loin de constituer

25. Boccace, I, 3,7-9: «Insuper, rex precipue, sciendum est his fictionibus non esse tantum unicum intellectum, quin imo dici potest potius polisenum, hoc est multiplicium sensum. Nam sensus primus habetur per corticem, et hic licteralis vocatus est; alii per significata per corticem, et hi allegorici nuncupantur. Et ut quid velim facilius assummatur, ponemus exemplum. Perseus Iouis filius figmento poetico occidit Gorgonem, et uictor euolauit in ethera. Hoc dum legitur per licteram hystorialis sensus prestatur. Si moralis ex hac lictera queritur intellectus, uictoria ostenditur prudentis in uicium, et ad uirtutem accessio. Allegorice autem si uelimus assummere, pie mentis spretis mundanis deliciis ad celestia eleuatio designatur. Preterea posset et anagogice dici per fabulam Christi ascensum ad patrem mundi principe superato figurari. Qui tamen sensus etsi uariis nuncupentur nominibus, possunt tamen omnes allegorici appellari, quod ut plurimum fit. Nam allegoria dicitur ab allon, quod alienum latine significat, siue diuersum, et ideo quot diuersi ab hystoriali seu licterali sint sensu, allegorici possunt, ut dictum est, merito uocitari. Verumtamen non est animus michi secundum omnes sensus enucleare fabulas que sequuntur, cum satis arbitrer unum ex pluribus explicasse, esto aliquando apponentur fortasse plures. » 
un carcan qui appauvrirait loriginalité de la fable païenne, inspire une interprétation plus dynamique et ouverte. Avec Boccace, l'allégorie n'est pas tenue de correspondre point par point au système extrêmement codifié de la quadruple exégèse. Elle devient une interprétation aux frontières beaucoup plus floues, consistant essentiellement à associer à une fiction plusieurs sens différents, anciens et modernes, que chaque nouveau lecteur peut enrichir de sa propre interprétation.

\section{Théologies chrétienne et varronnienne}

Cet apparent relativisme dans l'interprétation n'amène pas à dévaloriser les fictions des poètes, on l'a vu, mais au contraire à les hausser au rang des figures dont usent le Christ et ses apôtres. Interpréter les fables, selon Boccace, est un travail de théologien. Par ce terme, il ne désigne pas, tant s'en faut, les théologiens de son temps, pourvoyeurs d'une interprétation trop rigide et pourfendeurs de la poésie. La théologie qui intéresse Boccace est une théologie poétique, exprimée en figures ou fictions, que les hommes ont toute latitude d'interpréter diversement. On passe ainsi du paradigme de l'exégèse chrétienne à celui de l'interprétation polysémique des poètes païens, tout en conservant cette référence à la théologie. La théologie est ici comprise dans son sens étymologique, comme un discours sur le ou les dieux, quels qu'ils soient, paiens ou chrétiens. Il y a donc une redéfinition implicite de la tâche du théologien. Pour ce faire, Boccace se place sous le patronage de Varron, érudit latin du $\mathrm{I}^{\mathrm{er}}$ siècle avant J.C. Il s’adresse en ces termes à son dédicataire, qui n’est autre que le roi de Chypre et de Jérusalem:

Sane circa huiusmodi explicationes longe plus quam putes difficultatis et theologi hominis labor est, nam, dato, iuxta Varronis sententiam, ubi de divinis et humanis rebus multa descripsit, genus hoc theologie sit, quod mithicon seu, ut aliis placet et forte melius, physicon dicitur, et si plurimum ridende falsitatis habeat, multum tamen ad illam eliciendam artis exquirit ${ }^{26}$.

26. Boccace, Genealogia, Préface, I, 18 (Préface, I, 18). 
Assurément, concernant ce genre d'explication il y a beaucoup plus de difficultés qu'on ne pense et c'est un travail de théologien, d'autant que, suivant le sentiment de Varron qui a tant écrit sur les choses divines et humaines, la théologie dont il s'agit est celle qu'on appelle mythique ou, de l'avis peut-être meilleur de quelques autres, physique.

L'interprétation des fables relève selon Boccace d'un " genre de théologie » ("genus hoc theologiae ») bien particulier. Il s'agit de la théologie varronienne, qui a été transmise jusquà lui par l'intermédiaire notamment d'Augustin $^{27}$ et Tertullien ${ }^{28}$, qui expliquent ses fondements pour mieux la critiquer. D’après ces Pères de l'Église, Varron propose (ou reprend au juriste romain Scaevola) une tripartition de la théologie: « on appelle théologie mythique celle dont usent surtout les poètes, physique celle des philosophes, civile celle des peuples ${ }^{29}$. Boccace ne reprend pas explicitement cette tripartition. Il semble confondre les deux premières catégories, la théologie mythique et la théologie physique, et omettre la troisième, la théologie civile. La fusion des deux types de théologie mythique et physique s'inscrit tout naturellement dans le projet de Boccace: les fables des poètes sont polysémiques; elles peuvent donner lieu à des interprétations physiques car elles recèlent aussi un savoir qui relève de la philosophie naturelle. Il reste à comprendre pourquoi Boccace omet la troisième catégorie, la théologie civile. Dans la perspective varronienne, ce troisième aspect de la théologie est utilisé par les rois et gouvernants pour inciter leurs sujets à bien se comporter. Le meilleur exemple en est le roi Numa, tel qu'il est décrit dans l'Histoire de Rome de Tite-Live ${ }^{30}$ : le bon roi inspire

27. Voir notamment les livres IV, VI et VIII de la Cité de Dieu.

28. Tertullien, Ad nationes, livre II, cité par Jean Pépin, « La « théologie tripartite » de Varron: essai de reconstitution et recherche des sources ", dans Mémorial Gustave Bardy, Revue des études augustiniennes, 2, 1956, tome II, p. 265-294.

29. Augustin rapporte les conceptions de Varron dans la Cité de Dieu, VI, 5: «Mythicon appellant, quo maxime utuntur poetae; physicon, quo philosophi, ciuile, quo populi. »

30. Tite-Live, Histoire de Rome, I, 19, 4-5, traduction de Danielle De Clercq, Bruxelles, 2001 : « Il jugea que le moyen le plus efficace pour agir sur la masse inexpérimentée et inculte de ce temps-là était de lui inculquer avant tout la crainte des dieux. Comprenant qu'il ne pouvait se mettre au niveau de ses sujets sans recourir à un artifice relevant du merveilleux, Numa leur fit croire qu'il avait en pleine nuit des entretiens avec la déesse Égérie: "C’est sur son conseil à elle, leur disait-il, que j’institue des célébrations que les dieux apprécient tout particulièrement et que je prépose au culte de chaque dieu ses propres prêtres". » 
à son peuple la crainte des dieux et leur fait croire qu'il s'entretient avec la déesse Egérie pour accréditer les réformes qu'il est en train d'instaurer. Boccace ne mentionne pas, dans sa préface de la Genealogia, la dimension politique de la théologie: ce geste aurait probablement été beaucoup trop osé dans un passage où il harangue directement le roi en personne. Peutêtre le destinataire aura-t-il su lire entre les lignes. Boccace évoque par contre explicitement la fonction civile de la fable à la fin de son ouvrage. Au livre XIV, il rappelle l'anecdote de Memnius Agrippa, qui évita une guerre civile entre patriciens et plébéiens en racontant une fable, plus précisément l'apologue de l'estomac ${ }^{31}$. Boccace, s'il ne répète pas de manière explicite sa conception de la théologie tripartite, s'en inspire visiblement et s'en revendique en citant Varron, afin de souligner toute l'utilité que l'on peut tirer des fables des païens.

L'interprétation des fables dans la perspective de la théologie varronienne ${ }^{32}$ sera pleinement exploitée par les mythographes du $\mathrm{XvI}^{\mathrm{e}}$ siècle, tous épigones de Boccace. Celui-ci a donné une impulsion décisive, dont ses successeurs tirent toutes les conséquences: puisque les interprétations sont multiples et polysémiques, puisque les fables ne permettent pas d'accéder à un sens unique et originel, l'attention des mythographes plus tardifs se déporte sur les usages concrets et pratiques de la fiction au sein des sociétés humaines. Il ne s’agit plus de révéler la vérité contenue dans une fable, mais de montrer la multiplicité des interprétations que les hommes en ont faites. À partir de cette multiplicité, les mythographes étudient alors les différents usages des fables dans les sociétés antiques. Ils mêlent citations poétiques, interprétations physiques et considérations sur les rites que ces fables sont censées expliquer. Cette démarche

31. Boccace, Genealogia, XIV, 9: « Fabulis quippe, quas isti ob uocabulum ita despiciunt, non nunquam legimus incitatos insano feruore animos fuisse sedatos et in mansuetudinem redactos pristinam; ut puta, dum a Memnio Agrippa, grauissimo uiro, romana plebs a patribus dissidens a Sacro monte in patriam per fabulam reuocata est. »Cette anecdote célèbre est rapportée par Tite-Live, Histoire romaine, II, 16.

32. Cf. Bodo Guthmüller, "Formen des Mythenverständnisses um 1500 », in Die Allegorese des antiken Mythos, Vorträge, gehalten anlässlich des 31. Wolfenbütteler Symposions vom 28. September bis 1. Oktober 1992 in der Herzog-August-Bibliothek Wolfenbüttel, dir. Hans-Jürgen Horn et Hermann Walter, Wiesbaden, Harassowitz, 1997, p. 37-61. 
est proche de celle de la théologie tripartite, que Boccace évoquait au seuil de sa Genealogia, et dont les mythographes développent les implications.

Giraldi se réfère explicitement à la théologie varronienne au seuil de son De deis gentium historia (1548), en évoquant bien, cette fois, les trois dimensions poétique, physique et civile de cette tripartition ${ }^{33}$. Il évoque à de multiples reprises le rôle de Numa Pompilius dans l'institution de rites religieux bénéfiques à la société civile. Il étudie enfin tout spécialement les usages civiques liés aux anciennes fables dans le dernier des dix-sept syntagmes qui composent son ouvrage. Celui-ci consiste en un passage en revue des sacrifices, des temples, des sacerdoces et festivités, des expiations, des supplications, des lectisternes et autres cérémonies civiles et religieuses, permettant de comprendre l'usage des fables chez les divers peuples qui composent l'empire romain. Au début de l'ouvrage, il explique que les Anciens ont attribué aux dieux « diverses figures ${ }^{34} »$. Les dieux sont présentés comme des entités formées, façonnées par les hommes dans un but civique.

Sunt etiam qui tradant, gentes effingere solere suos deos, prout earum erant instituta et mores ${ }^{35}$.

Certains vont jusquà rapporter que les païens avaient l'habitude de se façonner leurs dieux en fonction de leurs institutions et de leurs mœurs.

Giraldi emploie l'expression significative " effingere deos » : on façonne des dieux comme on façonne des fictions ou des fables, théologie civile et théologie des poètes ont des champs d'application différents mais relèvent d'une même activité de façonnement.

Pictorius aussi, comme Giraldi et Boccace, se réfère à Varron ${ }^{36}$ dès l'ouverture des Apotheoseos deorum libri (1558). Il analyse ensuite la

33. Giraldi, De Deis gentium historia, Bâle, Oporin, 1548, p. 18: « His commode afferre possumus eam theologia diuisionem, quam Marcus Varro in libris Antiquitatum divinarum humanarumque rerum fecisse legimus apud Augustinum: mythicon scilicet, hoc est fabulosum, physicon, id est naturale, et

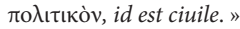

34. Giraldi, De Deis gentium historia, Bâle, 1548, p. 19: «attribuerunt... diuersis figuris ».

35. Giraldi, De Deis gentium historia, Bâle, 1548, p. 19.

36. Pictorius, Apotheoseos deorum libri, Bâle, Nikolaus Brylinger, 1558, p. 1. Pour une étude plus détaillée de la référence à Varron dans l'œuvre de Pictorius, ainsi qu'une analyse du titre selon la première (Apotheseos libri) et la seconde émission (Apotheoseos libri), voir ma thèse de doctorat. 
manière dont certaines fables déterminaient le fonctionnement des sociétés antiques. Il s'intéresse notamment aux prêtres et aux sacrifices ${ }^{37}$, à la coutume consistant à enchaîner les statues des dieux tutélaires lors du siège d'une ville ${ }^{38}$, aux fables justifiant la localisation d'un temple dans un lieu précis $^{39}$, aux serments inviolables faits devant la statue de Jupiter Lapis ${ }^{40}$. Il revient, comme Giraldi avant lui, sur l'usage que fit Numa Pompilius de la mythologie pour instaurer le droit et la paix, en ordonnant que Janus soit représenté et honoré sous la forme d'une « figure ${ }^{41}$ » dont il a lui-même décidé. Dans l'épître dédicatoire de sa Theologia mythologica publiée quelques années plus tôt (1532), Pictorius souligne d'emblée la fonction civique des fables des Anciens:

Quique nec illud perspicere quiuit, non poetas modo, sed et clarissimas ciuitatum quandoque fabulis usas, et legumlatores, qui imperitiorem multitudinem hac ratione ad bonum saepius elicuisse deprehenduntur ${ }^{42}$.

Il [le détracteur des fables] ne comprit pas que non seulement les poètes, mais aussi les cités les plus illustres usèrent jadis des fables, ainsi que les législateurs, que l'on surprend à attirer la foule, par ce moyen, constamment vers le bien.

Le titre de l'ouvrage, Theologia mythologica, se réfère manifestement à la « theologia mythica » de la théologie tripartite varronienne. Pictorius poursuit le travail que Boccace appelait de ses vœux. Il explique les anciennes fables comme une théologie, dont les implications sont à la fois poétiques, civiles et physiques. Il expose tour à tour les différentes figures que constitue chacun des dieux paiens, en suivant l'ordre des planètes auxquels les dieux correspondent dans le système astronomique ptolémaïque: d'abord Saturne, puis Jupiter, Mars, Apollon c'est-à-dire le Soleil, Vénus, Mercure, Diane c'est-à-dire la Lune. Son traité mythographique participe ainsi de la théologie physique: il rapporte les théories

37. Pictorius, Apotheoseos deorum libri, p. 2.

38. Pictorius, Apotheoseos deorum libri, p. 4.

39. Pictorius, Apotheoseos deorum libri, p. 5.

40. Pictorius, Apotheoseos deorum libri, p. 7.

41. Pictorius, Apotheoseos deorum libri, p. 73.

42. Pictorius, Theologia mythologica, épître dédicatoire, p. $3 \mathrm{v}^{\circ}$. 
et interprétations des philosophes sur la nature et le cosmos. Ainsi, les mythographes usent toujours de l'allégorie pour interpréter les fictions mythologiques, mais ils interrogent désormais le besoin qu'ont les hommes de se créer des figures, des fictions, et la manière dont ils en usent pour créer à la fois de beaux poèmes, des interprétations du monde et des systèmes d'organisation de la vie civile. Ils s'intéressent d'avantage, désormais, à la dimension pragmatique des fables et figures, à leur usage dans les sociétés humaines plus que dans la relation entre l'homme et un Dieu spécifiquement chrétien.

Certes, les mythographes rappellent toujours, dans leurs chapitres liminaires, que toutes les figurations païennes renvoient, en dernier recours, $\mathrm{au}$ " vrai Dieu ». Mais leur intérêt porte désormais sur la diversité des figures, bien plus que sur l'unicité du signifié qu’elles sont censées désigner. Il est difficile de savoir si cette attitude témoigne d'un certain relativisme sceptique à l'égard des religions. Les mythographes, bien sûr, ne s'exposent pas dans leurs publications. Giraldi, qui a publié sa mythographie à Bâle plutôt qu'en Italie, mentionne rapidement l'existence d'un danger, sans développer davantage:

Et cum [Deus] unus sit, nefas est existimare, alios apud alios esse deos, seu barbaros illos, seu Graecos atque Romanos. Sed uti Sol, Luna, Caelum, Terra, Mare, communia sunt omnibus, licet aliis apud alios nominibus appellentur: ita eadem ratione unus est Deus, cuius ubique uis est et prouidentia, omnia alit, sustinet, exornat: alii tamen apud alios sunt ritus legibus instituti, aliae caeremoniae, alia nomina, alia symbola, obscure quidem a primis auctoribus condita et consecrata, uni tamen supremo et praepotenti, quae aptari et conuenire debent, quorum omnium interpretationem et explicationem non facile, nec sine periculo quilibet disquirere, uel praesumere potest ${ }^{43}$. Comme il n'y a qu'un seul Dieu, il est sacrilège de penser qu'il y a d'autres dieux chez d'autres peuples, soit barbares, soit grecs et romains. Mais de même que le Soleil, la Lune, le Ciel, la Mer sont communs à tous, bien qu'ils soient appelés par d'autres noms chez d'autres peuples, de même, selon le même principe, il y a un Dieu, dont la force et la providence, où quelles soient, nourrissent, soutiennent, ornent tout; toutefois les rites,

43. Giraldi, De Deis gentium historia, Bâle, Oporin, 1548, p. 1-2. 
institués par les lois, sont différents chez les uns et chez les autres, différents les cérémonies, les noms, les symboles, mystérieusement cachés et consacrés par les premiers fondateurs; mais c'est à un seul Dieu suprême et tout-puissant que tout cela doit cependant être attaché et convenir, tout cela dont le premier venu ne peut s'enquérir ni présumer facilement ni sans danger.

Giraldi concentre nettement son enquête sur l'institution ( instituti»), la fondation ( « condita ») des rites et des symboles. Il n'entend plus interpréter les fables pour se rapprocher de Dieu, comme le faisait Boccace. Il s'intéresse bien plus aux hommes, à la manière dont ils fabriquent un système de figures pour assurer le respect des lois. On voit ainsi émerger, très progressivement, une perspective qui deviendra celle de l'histoire des religions ${ }^{44}$. La figure, mode d'expression privilégié de la vérité divine dans la tradition médiévale, est de plus en plus étudiée, au $\mathrm{XVI}^{\mathrm{e}}$ siècle, comme un mode d'expression régulateur des sociétés humaines. Plus que la figure, c'est la diversité des modes de figuration, adaptés à chaque lieu et à chaque société, qui motive désormais l'enquête mythographique.

Natale Conti rappelle lui aussi, dans les chapitres liminaires, que " non seulement les narrations fabuleuses, mais aussi les images fabuleuses et les peintures ${ }^{45}$ » ont été introduites par les sages pour inciter le peuple ignorant à bien se comporter. Figuration des poètes et figuration des législateurs relèvent, à l'origine, d'une seule et même action, qui fonde les civilisations. Mais c'est le savoir-faire des poètes que Conti se plaît à nous faire partager. Ses Mythologiae libri proposent des citations poétiques beaucoup plus longues, plus développées, plus nombreuses que les autres traités. Conti, poète à ses heures, insère également des vers de son propre cru. Même si la fable suscite toujours des interprétations allégoriques, même si elle recèle d'anciens secrets, elle est avant tout donnée à lire, à savourer, à admirer. La citation poétique prend de plus en plus

44. Cf. Giovanni Ricci, « L’altro Giraldi. Lilio Gregorio alle origini del relativismo culturale », Giovan Battista Giraldi Cinzio gentiluomo ferrarese, dir. Paolo Cherchi, Micaela Rinaldi e Mariangela Tempera, Firenze, Olschki, 2008.

45. Natale Conti, Mythologiae, siue explicationum fabularum libri X, édition consultée: Venise, Wechel, 1581, ch. 2, «De fabularum utilitate », p. 3, lignes 19-25. 
d’ampleur, quantitativement, par rapport à l'interprétation. Si elle dissimule toujours un autre sens à interpréter, l'écorce n’est plus déconsidérée. Loin de ne constituer qu'un simple prétexte pour parvenir à un autre degré de vérité, elle est désormais au centre de lattention.

La notion de figure évolue ainsi de manière décisive entre l’œuvre de Dante et celle de Conti. Dans la Divine Comédie, Dante se représente comme un homme qui a vu Dieu. C'est cette vision qui éclaire rétrospectivement les figures qu'il rencontre sur sa route. La description qu'il en fait vise à transmettre aux autres hommes cette expérience privilégiée. Boccace, lui, ne contemple pas Dieu face à face. Il se place seulement sous sa protection, le supplie de lui donner l'inspiration. La figure, chez Boccace, rapproche l'homme de la connaissance divine, mais elle ne l'y mène pas en droite ligne. Les mythographes du Xvi ${ }^{\mathrm{e}}$ siècle soulignent encore plus cet éloignement. On voit ici à quel point la caractérisation de la figura par Auerbach est efficace pour comprendre Dante, précisément dans ce qui le différencie des auteurs ultérieurs. Tandis que, chez Dante, la figure inscrit les êtres dans la double temporalité du Jugement Dernier, elle est davantage la marque, aux yeux des mythographes du Xvi ${ }^{\mathrm{e}}$ siècle, de l'immanence de l'humain. Chaque peuple, chaque époque façonne, avec les instruments qui sont les siens, les figures censées le mener au divin. Cette évolution est intimement liée à celle de la notion de théologie, qui, au contact des lettres anciennes, se voit sécularisée et recontextualisée. S'il n'y a toujours, aux yeux des mythographes de la Renaissance, qu'un seul Dieu chrétien, la diversité des discours qui y mènent présente un intérêt en tant que tel. La référence à Varron correspond à un recentrement de la théologie, devenue poésie, du divin vers l'humain. C'est donc désormais la figuration, plus que la figure, lélaboration des hommes, plus que la trace de Dieu, qui fascine les lettrés d'un siècle en proie au vertige de l'interprétation. Le poète, s'il est inspiré par Dieu, est avant tout au service de la cité. Les trois aspects de la théologie varronienne s'entrelacent sous sa plume. Le rôle du mythographe est alors de savoir interpréter l'utilité de 
ces fables, plus que leur vérité. Si la figure renvoie vers un sens originel de plus en plus éloigné et hors d'atteinte, la figuration et la fiction, quant à elles, offrent des outils pour mieux vivre en société. De la mythographie au projet utopique se sont ainsi tissés des liens qui restent encore à démêler. Comme l'a montré Marie Madeleine Fontaine ${ }^{46}$, la mythographie de Giraldi a joué un rôle décisif dans lélaboration du roman Alector et de son héros législateur. Il s'agit chaque fois de comprendre la notion de mythe fondateur, dans un cas par une enquête sur les fictions du passé, dans l'autre par une fiction nouvelle, élaborée à nouveaux frais. Une plus vaste étude reste à mener sur l'enjeu politico-juridique commun à ces deux types de texte qui nont probablement pas manqué de s'influencer. Ce sera, je l'espère, l'objet d'un prochain chantier.

46. Marie Madeleine Fontaine, introduction à Barthélémy Aneau, Alector ou Le Coq, histoire fabuleuse, Genève, Droz, 1996. 